\section{La intervención del pediatra en el niño con labio y paladar hendido}

\section{The intervention of the pediatrician in the child with cleft lip and palate.}

Lombardo-Aburto $\mathrm{E}$

\section{INTRODUCCIÓN}

Las malformaciones congénitas son defectos estructurales presentes en el nacimiento. ${ }^{1}$ Las fisuras labio-alvéolo-palatinas tienen una alta incidencia que se presenta en uno de cada 750 recién nacidos vivos, ${ }^{2}$ por lo que están entre las malformaciones más frecuentes y mejor conocidas. ${ }^{3}$ El labio y paladar hendido son anomalías que comúnmente están asociadas y constituyen las malformaciones congénitas más frecuentes de la cabeza y el cuello. ${ }^{4}$ Se define como labio leporino o hendido, fisura labial o queilosquisis al defecto facial que involucra el cierre incompleto del labio, unilateral, bilateral o medial, generalmente lateral a la línea media. Se define como paladar hendido, palatosquisis, o fisura palatina al defecto palatino en la línea media que comunica con fosas nasales y cavidad oral.

\section{EPIDEMIOLOGÍA}

La incidencia de labio leporino es mayor en los varones y la de paladar hendido en las mujeres; la relación es de 7:3, afectando más a varones. La frecuencia es $21 \%$ del labio hendido aislado, $33 \%$ de la fisura palatina aislada y $46 \%$ de ambas lesiones simultáneas, también es más frecuente el labio hendido unilateral izquierdo. $^{2}$

De los pacientes que padecen labio y paladar hendido en $25 \%$ de los casos se conoce la causa. En $75 \%$ de los casos la causa es multifactorial y en 20-25\% de los casos existe algún antecedente familiar. ${ }^{2}$ El antecedente familiar con labio y paladar hendido aumenta el riesgo de heredarle de 4 a $20 \%$. El mayor riesgo que
Médico Pediatra, adscrito a la Consulta Externa. Instituto Nacional de Pediatría, México.

Recibido: 3 de marzo del 2017

Aceptado: 15 de marzo del 2017

Correspondencia Esther Lombardo Aburto elombardoaburto@yahoo.com.mx

Este artículo debe citarse como Lombardo-Aburto E. La intervención del pediatra en el niño con labio y paladar hendido. Acta Pediatr Mex. 2017;38(4):267-273. 
ocurra es entre la cuarta y octava semana de gestación. Un 5\% de estas dos malformaciones forman parte de otros síndromes (Figura 1). Los factores asociados con estas malformaciones congénitas se pueden reunir en dos grandes grupos: ambientales y genéticos.

\section{Ambientales}

Pueden ser físicos, químicos o biológicos y que, por alterar el desarrollo embriológico causando malformaciones, se denominan teratógenos. Entre ellos la edad de los padres pues el riesgo se incrementa a mayor edad por arriba de los 30 años; según Habib $^{5}$ la estación del año, el país de residencia, la raza; infecciones ma- ternas, principalmente virales; desnutrición, abortivos (aminopterina); madres epilépticas que consumen anticonvulsivos durante el primer trimestre del embarazo (difenilhidantoina): falta o deficiencia en la ingesta de ácido fólico y ácido retinóico durante el embarazo; alcohol, tabaquismo materno, plomo, antibióticos, radiaciones ionizantes, pesticidas.

\section{Genéticas}

Dependiendo del origen racial las malformaciones son variables, desde 1 en 500 nacimientos en poblaciones asiáticas, a 1 en 2,500 en raza negra y 1 en 1,000 entre caucásicos, hispánicos y latinos. ${ }^{6}$ Se reporta una alta frecuencia de

\section{¿Cuándo hay mayor riesgo de que aparezcan?}

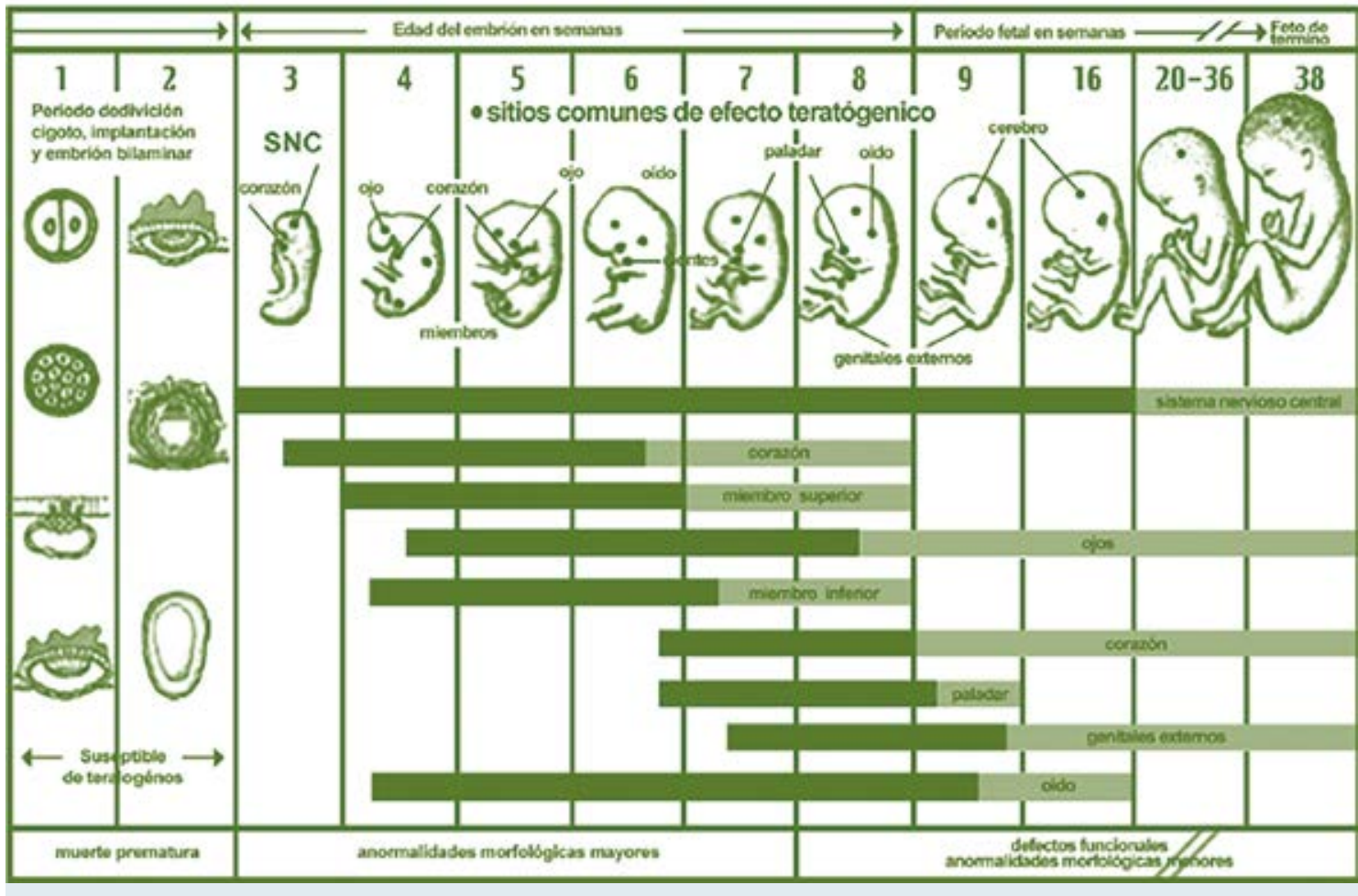

Figura 1. Etapas de desarrollo embrionario. Tomado de: Centro Nacional de Equidad de Género y Salud Reproductiva. SSA. Prevención, tratamiento, manejo y rehabilitación de niños con labio y paladar hendido, 2006. 
fisuras labio-palatinas en los siguientes países de Sudamérica: Bolivia, Ecuador y Paraguay. En México, los estados con más incidencia son Oaxaca, Chiapas, Guerrero, Puebla y Veracruz. González-Osorio y sus colaboradores reportaron, en un estudio ecológico en México durante el período del 2003-2009, un total de 10,573 nuevos casos de labio y paladar hendido, con un mayor número de casos en el año 2004. ${ }^{7}$ En una casuística de 10 años de labio y paladar hendido, con un total de 376 casos, realizado en el Hospital Universitario de Nuevo León, en Monterrey, no detectaron como factores de riesgo la edad de los padres, la ingesta de medicamentos ni las enfermedades crónicas. ${ }^{4}$

\section{SINTOMATOLOGÍA}

Las fisuras labio-alvéolo-palatinas pueden implicar la deformidad de 4 estructuras diferentes: el labio, el proceso alveolar, el paladar duro y el paladar blando, con la posibilidad que la alteración sea unilateral o bilateral, completa o incompleta.

El labio leporino puede tener varios grados o tipos: a) una pequeña muesca en el borde labial superior; b) fisura labial aislada con escasa alteración maxilar, pero habitualmente con mala implantación y mala oclusión dentaria y deformidad del ala nasal como alteraciones secundarias de la secuencia, lo mismo que la fisura palatina, c) formas graves de la queilosquisis y queilognatosquisis con gran deformidad bucal: labio leporino completo y bilateral.

En la fisura palatina las gradaciones son: a) forma grave $(10 \%)$, con fisura labial bilateral y hendidura palatina total, tanto del paladar óseo como blando; b) tipo más frecuente $(40 \%)$ con labio leporino total unilateral y fisura palatina total; c) fisura palatina aislada (30\%) con intensidad variable, pero con participación maxilar; d) fisura del paladar blando (velopalatina) o con hendiduras mucosas (20\%); úvula bífida aislada. Si la hendidura es total, el neonato y el lactante tendrán dificultad para la succión y deglución.

Más tarde se agregarán los trastornos terciarios de la secuencia: otitis media, sordera, problemas del lenguaje.

\section{COMPLICACIONES}

Inmediatas

Dificultades en la alimentación. Debido al problema para la succión, por anomalías del paladar hendido, se condiciona una detención en peso o talla llegando a una desnutrición.

Broncoaspiración. Por la comunicación entre el paladar y narinas; por mal manejo de secreciones nasofaringeas, leche o ambas.

\section{Mediatas}

Alteraciones auditivas. Infecciones o hipoacusia debido a una disfunción y horizontalización de las trompas de Eustaquio, que conecta el oído medio con la faringe.

\section{Tardías}

Alteraciones del lenguaje. Retardo o deficiencia en el lenguaje debido a una mala implantación de los músculos del paladar lo que reduce o altera la función de audición.

Problemas odontológicos. Puede presentarse mal posición dentaria.

Problemas emocionales. El impacto que causa en los padres un hijo con malformación provoca culpabilidad o rechazo; lo que se traduce en un retardo tanto psicoemocional como en el manejo terapéutico. De igual manera sucede para el paciente al ingresar a la escuela o entre otros 
familiares condicionando falta de aceptación o bullying.

\section{MANEJO INTEGRAL}

Dada la complejidad de la deformidad maxilofacial se requiere de un manejo multidisciplinario en donde intervengan diferentes áreas:

1. Áreas quirúrgicas: cirugía maxilofacial, cirugía plástica y anestesia.

2. No quirúrgicas: estomatología, odontopediatría, otorrinolaringología, audiología, foniatría, genética, pediatría y psicología.

El tratamiento quirúrgico primario busca lograr un cierre óptimo, una perfecta función y buen resultado estético, por lo cual es necesario que al paciente se le refiera a la unidad especializada prácticamente desde los primeros días de vida. Se requieren un mínimo de 4 eventos quirúrgicos por caso. El primero a los tres meses de edad para cierre de labio y plastia de punta nasal; el segundo entre los 12 y 18 meses de edad para el cierre del paladar y faringoplastia; el tercero entre los 6 y 8 años de edad con injerto óseo alveolar; el cuarto se efectúa después de los 14 años y es la cirugía estética facial (rinoseptumplastia, mentoplastia, etc.).

\section{CÓMO Y CUÁNDO DEBE ACTUAR EL PEDIATRA}

El pediatra debe formar parte del grupo y manejo multidisciplinario de labio y paladar hendido ya que, como otras malformaciones, ésta puede ser prevenible, diagnosticada y manejada oportunamente; por lo tanto, el pediatra debe actuar en los 3 niveles de prevención:

Prevención primaria. Dirigida a la promoción de la salud y protección específica. Se debe ejercer prevención desde la atención médica de la mu- jer embarazada en coordinación con el médico gineco-obstetra, en los siguientes puntos:

1. A toda mujer en edad reproductiva; esto incluye desde la adolescencia: la administración diaria de ácido fólico de 400 mcg/día o 0.4 mg, especialmente en la etapa periconcepcional (3 meses previos al embarazo y hasta la semana 12 de

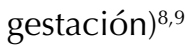

2. Detección oportuna de factores de riesgo relacionados con el medio ambiente y de la madre, para implementar medidas de prevención dirigidas a la eliminación o modificación del factor o factores causales.

3. Promoción de la salud en todos los niveles de atención, pero principalmente en la atención primaria en coordinación con el personal de enfermería, trabajo social y promotores de la salud, difundiendo toda la información relacionada con labio y paladar hendidos implementando pláticas, difusión de la información a través de otros medios de comunicación como radio, televisión, internet. También con trípticos, carteles, campañas, etc.

4. Sensibilizar a la mujer embarazada sobre la importancia de asistir puntal y tempranamente a sus consultas obstétricas, cumpliendo con un mínimo de 5 consultas según lo establecido por la Organización Mundial de la Salud.

5. Consejo genético informando a los padres del riesgo de otros casos con labio y paladar hendidos para evitar la presentación de casos nuevos en familias con antecedentes o factores de riesgo genéticos reconocidos.

Prevención secundaria. Dirigida al diagnóstico temprano y tratamiento oportuno. 
1. Seguimiento obstétrico de toda mujer embarazada para la detección temprana de alguna patología, entre ellas, malformaciones congénitas como labio y paladar hendido.

2. Seguimiento en conjunto con el pediatra para el diagnóstico oportuno y el conocimiento de los antecedentes maternos.

3. Revisión integral del recién nacido por el pediatra para detectar otras malformaciones congénitas y su manejo integral.

4. Clasificar el tipo de labio y paladar hendido y su manejo integral dirigido a una adecuada alimentación y el uso de placa obturadora si así lo requiere el paciente.

5. Alertar a los padres sobre el riesgo de bronco aspiración y manejo inadecuado de secreciones en este tipo de niños.
6. Coordinación con los médicos especialistas en el manejo de labio y paladar hendido para su corrección quirúrgica y manejo por los especialistas afines.

7. Seguimiento pediátrico dirigido a prevenir complicaciones óticas, dentales, fonación y psicológicas en conjunto con los especialistas de cada área.

Prevención terciaria. Dirigida a la rehabilitación y manejo de las complicaciones. Canalizar oportunamente al paciente para recibir atención médica por los servicios de otorrinolaringología, orto-odontopediatria, psicología y terapia de rehabilitación por los servicios de foniatría, audiología y así disminuir complicaciones y secuelas (Cuadro 1).

\section{EVOLUCIÓN}

Dependerá de los trastornos digestivos, respiratorios y malformaciones asociadas de cada paciente.

Cuadro 1. Cronograma terapéutico para pacientes con labio y paladar hendido (continúa en la siguiente página)

\section{Edad del paciente $\quad$ Manejo quirúrgico y de rehabilitación por especialista}

Recién nacido hasta primer mes de vida
Revisión clínica completa por el pediatra, detectar otras malformaciones congénitas e historia clínica integral.

Orientar a los padres sobre su alimentación indicando seno materno o en su defecto utilizar alimentador con jeringa.

Indicar a los padres que "el chupón de aleta" NO es útil.

Presentación del niño en la clínica de labio y paladar hendido y su clasificación.

Tratamiento ortopédico-ortodóntico para colocación de placa ortopédica obturadora si es necesario. Esta placa tiene varias finalidades:

-por medio de las presiones dirigidas mejorar la arcada dental y erupciones dentarias adecuadas en etapas tardías

-evitar el paso de líquidos y alimentos sólidos a la nariz y prevenir broncoaspiración -mejorar su alimentación y prevenir detención de peso y talla

Enseñar a la madre la higiene oral y de la placa obturadora.

Una semana después de la colocación de la placa ortopédica obturadora (ya que el binomio madrebebé estén adaptados a la misma) se coloca el conformador nasal con la finalidad de remodelar el cartílago nasal. Se utiliza hasta los 3 meses de edad que corresponde a la edad de la queiloplastia. Audiología para realizar tamiz auditivo.

Foniatría para iniciar con los dispositivos fisiológicos (succión, deglución, masajes orofaciales y estimulación multisensorial).

Atención de los padres por psicología y genética. 
Cuadro 1. Cronograma terapéutico para pacientes con labio y paladar hendido (continuación)

\begin{tabular}{|c|c|}
\hline Edad del paciente & Manejo quirúrgico y de rehabilitación por especialista \\
\hline 3 meses & $\begin{array}{l}\text { Cierre de la fisura labial y plastía de punta nasal. } \\
\text { Seguimiento pediátrico para control de alimentación, desarrollo psicomotor integral e inmunizaciones. } \\
\text { Foniatría estimulación de balbuceo y continuación de masajes orofaciales y estimulación multisensorial } \\
\text { Audiología y otorrinolaringología: realizar impedanciometría. }\end{array}$ \\
\hline 6 meses & Foniatría: iniciar con onomatopeyas, esquema corporal. \\
\hline $12-18$ meses & $\begin{array}{l}\text { Cierre de paladar y faringoplastia. } \\
\text { Foniatría: estimular primeras palabras. } \\
\text { Revisión por el otorrinolaringología, prevenir complicaciones óticas como infecciones o hipoacusia } \\
\text { por la disfunción de la trompa de Eustaquio y horizontalización del conducto auditivo. } \\
\text { Control por estomatología de la erupción dental. } \\
\text { Continuar seguimiento por psicología y pediatría. } \\
\text { Intervención de foniatría para terapia del lenguaje al cierre del paladar para aprender a usar el músculo } \\
\text { del velo del paladar una vez que ha sido aproximado a su sitio anatómicamente adecuado. }\end{array}$ \\
\hline 2 años & $\begin{array}{l}\text { Seguimiento por estomatología de la erupción dental. } \\
\text { Ortopedia: continuar medidas de ortopedia funcional. } \\
\text { Seguimiento por los servicios de otorrinolaringología, pediatría y psicología. } \\
\text { Foniatría: praxias de lengua, labios y velosoplo. }\end{array}$ \\
\hline 3 años & $\begin{array}{l}\text { Inicia terapia intensiva del lenguaje y corregir los errores de pronunciación y seguimiento de instruc- } \\
\text { ciones. } \\
\text { Seguimiento pediátrico, esquema de vacunación completo y prevención de infecciones respiratorias. }\end{array}$ \\
\hline $4-6$ años & $\begin{array}{l}\text { Comienza etapa escolar } \\
\text { Vigilancia por psicología para adaptación escolar y prevención de bullying. } \\
\text { Manejo integral con los padres. }\end{array}$ \\
\hline$<6$ años & Foniatría conceptos prepedagógicos y estructuración del lenguaje. \\
\hline 6-8 años & $\begin{array}{l}\text { Injerto óseo alveolar. } \\
\text { Se debe realizar "antes de la erupción del canino permanente". } \\
\text { En caso necesario corrección secundaria de labio, paladar y nariz. } \\
\text { Intervención foniátrica y auditiva intensa. } \\
\text { Seguimiento por psicología, pediatría y ortopedia. } \\
\text { Foniatria para estimular los dispositivos básicos del aprendizaje. } \\
\text { Proceso de lectoescritura. }\end{array}$ \\
\hline 8-12 años & $\begin{array}{l}\text { Continuar el tratamiento de ortopedia funcional e inicio de ortodoncia si es necesario. } \\
\text { Vigilancia por el pediatra del inicio de su etapa puberal. } \\
\text { Seguimiento por psicología y foniatría. }\end{array}$ \\
\hline $\begin{array}{l}14 \text { años en ade- } \\
\text { lante }\end{array}$ & $\begin{array}{l}\text { Corrección de cirugía estética facial (nariz y si es necesario mentón, pómulos y mejillas). } \\
\text { Medidas protésicas y ortodoncia. } \\
\text { Seguimiento pediátrico en su etapa puberal. } \\
\text { Terapia intensiva por psicología durante la etapa de adolescencia. } \\
\text { Seguimiento por foniatría. }\end{array}$ \\
\hline
\end{tabular}

Nota: todas las medidas de ortodoncia-ortopedia van dirigidas a la preparación del injerto óseo alveolar. En todas la edades se debe dar seguimiento por todos los especialista que integran la clínica de labio y paladar hendidos; sin embargo, en el cronograma se hace mención a los aspectos más importantes.

\section{CONCLUSIONES}

La intervención del pediatra en el manejo del paciente con labio y paladar hendido es fundamental ya que es quién tiene el primer contacto con estos pacientes, desde la atención del recién nacido en las unidades tocoquirúrgicas o durante las primeras semanas de vida en la consulta pediátrica. 
Lombardo-Aburto E. Intervención pediátrica en labio y paladar hendido

El pediatra debe formar parte del grupo y manejo multidisciplinario de labio y paladar hendido ya que, como otras malformaciones, esta puede ser prevenible, diagnosticada y manejada oportunamente; por lo tanto, el pediatra debe actuar en los 3 niveles de prevención. Debe conocer el manejo integral de estos pacientes, incluyendo los tiempos quirúrgicos, la coordinación con las subespecialidades involucradas para una adecuada atención oportuna y la evolución satisfactoria con la prevención de complicaciones.

\section{REFERENCIAS}

1. Coiffman. F. Texto de cirugía plástica, reconstructiva y estética. La Habana: Editorial Científico-Técnica: 1986.

2. Centro Nacional de Equidad de Género y Salud Reproductiva. SSA. Prevención, tratamiento, manejo y rehabilitación de niños con labio y paladar hendido, 2006.

3. Corbo R.MT, Marimón TME. Labio y paladar fisurados. Aspectos generales que se deben conocer en la aten- ción primaria de la salud. Rev. Cubana Med gen Inter. 2001;17(4):379-85.

4. Jones MC. Facial clefting: etiology and developmental patogenesis. Clin Plast Surg 1993;20:599-611.

5. Ortega A. Deformidades craneofaciales, labio y paladar hendido o ambos. Pract Odont 1996;12:47-80.

6. Quijano MC, Rivas J, Salas I, Salazar M, Sánchez B, Sierra $\mathrm{CH}$. Aspectos sociodemográficos y clínicos de labio leporino y paladar fisurado en una población del suroccidente colombiano. Revista de la Facultad de Ciencias de la Salud Universidad del Cauca. 2009;11:25-3.

7. C.A. González-Osorio, C.E. Medina -Solis, A.P. PontigoLoyola y cols. Estudio ecológico en México (2003-2009) sobre labio y/o paladar hendido y factores sociodemográficos, socioeconómicos y de contaminación asociados. An Pediatr (Barc) 2011;74(6):377-387.

8. Valdés-Hernández J, Canún-Serrano S, Reyes-Pablo $A E$, Navarrete-Hernández E. Mortalidad por defectos en el cierre del tubo neural en menores de 5 años de edad en México de 1998 a 2006. Salud Publica Mex. 2010;52:341-9.

9. Bedón RM, Villota GLG. Labio y paladar hendido: tendencias actuales en el manejo exitoso. Archivos de Medicina 2012;12:107-119. 